\title{
Finding the sweet spot: Titrating unfractionated heparin in children after cardiac surgery to prevent thrombosis and minimize bleeding
}

\author{
Leslie Raffini, MD, MSCE, ${ }^{a}$ and M. Patricia Massicotte, MSc, MD, MHSc ${ }^{b}$
}

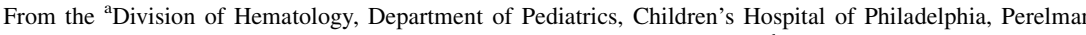
School of Medicine at the University of Pennsylvania, Philadelphia, Pa; and ${ }^{\mathrm{b}}$ Division of Cardiology, Stollery Children's Hospital, University of Alberta, Canada.

Disclosures: L.R. is a consultant to Bayer, HEMA Biologics, CSL Behring, and Genentech. None of these activities are relevant to this manuscript or editorial. M.P.M. is a consultant to the National Institute of Health/National Heart Lung Blood Institute, Bayer GmBh, and Xeltis BV/AG. None of these activities are relevant to this manuscript or editorial.

Received for publication March 3, 2018; accepted for publication March 7, 2018; available ahead of print April $12,2018$.

Address for reprints: M. Patricia Massicotte, MSc, MD, MHSc, 3-539 ECHA, 11405-87 Ave NW, Edmonton, Canada T6G 1C9 (E-mail: mm41@ualberta.ca).

J Thorac Cardiovasc Surg 2018;156:353-4

$0022-5223 / \$ 36.00$

Copyright (c) 2018 by The American Association for Thoracic Surgery

https://doi.org/10.1016/j.jtcvs.2018.03.043
}

Titration of unfractionated heparin (UFH) in children remains a challenge. In the past, the partial thromboplastin time (PTT) has been the primary assay to monitor "therapeutic treatment doses of UFH." 1 The limitations of the PTT as a single monitoring assay have become increasingly appreciated, and incorporation of the heparin anti-factor Xa (anti-Xa assay), a more direct measure of the anticoagulant effect, has grown. ${ }^{2}$ The correlation between PTT and anti-Xa in children receiving UFH is poor and is reconfirmed by this study (Figure 1). ${ }^{3}$ In most cases, the PTT is higher than in adults for a given anti$\mathrm{Xa}^{3}$ Nair and colleagues ${ }^{4}$ developed a protocol for postoperative UFH management in children after cardiac surgery using both the anti-Xa and PTT. A ceiling of 100 seconds for the PTT was used as a stop point for increasing UFH irrespective of the anti-Xa. In this prospective study, 87 patients managed with a standard UFH nomogram (PTT only) were compared with 116 patients treated with this new protocol. The primary finding was a reduction in clinically relevant bleeding events, with no increase in thrombosis, suggesting that this approach may be getting closer to the sweet spot for UFH. The rate of major bleeding in each group was the same.

Children treated with the new protocol had higher average PTTs and heparin doses. Compliance with new protocol was $81 \%$, compared with only $58 \%$ in the group treated using the standard PTT-only protocol. Although the number of patient-hours spent above the "therapeutic range" decreased, the upper PTT limit was 85 seconds for the PTT only group and 100 seconds for patients followed with both PTT and anti-Xa (unless the anti-Xa was $>0.7$ ). Thus, increasing both the maximum PTT and the protocol compliance (more frequent laboratory tests and dose adjustments) likely contributed to fewer patient hours above the therapeutic range, and the contribution of the anti-Xa assay to the reduction of bleeding

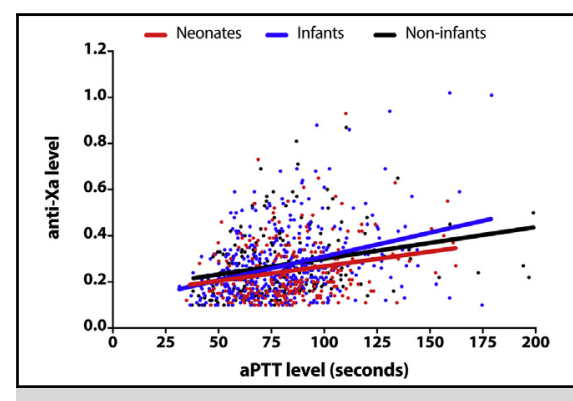

Is this the "sweet" spot? Anti-Xa versus PTT5: poor correlation but different information.

Central Message

Despite poor correlation, combined use of the PTT and anti-Xa assays may bring us closer to the "sweet spot" of UFH therapy in children postsurgery, preventing thrombosis while minimizing bleeding.

See Article page 343.

events remains unclear. Nonetheless, inclusion of a PTT upper limit to prevent UFH increases in children with low anti-Xa levels is a novel contribution to existing guidelines and would be expected to reduce bleeding compared with patients monitored using only the anti-Xa assay. Thrombosis rates in patients titrated using this protocol were not increased, suggesting that lower anti-Xa values in children may be effective.

Ultimately, it is encouraging that implementation of a standardized protocol for UFH, incorporating both PTT

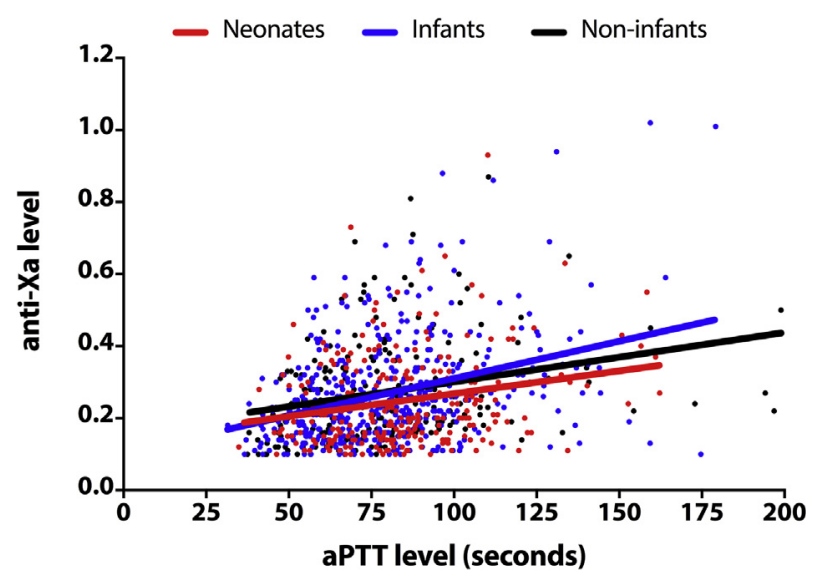

FIGURE 1. Is this the "sweet" spot? Anti-Xa versus PTT5: poor correlation but different information. Reprinted with permission. 
and anti-Xa, improved both compliance and reduced bleeding in this cohort. The generalizability of this protocol remains to be seen, and several issues warrant consideration: (1) This protocol was studied only in postoperative cardiac cases to prevent thrombosis and (2) coagulation assays between laboratory tests are highly variable; consequently absolute values of both PTT and anti-Xa from this single-center study may not correlate well with those in other centers. ${ }^{5}$ However, the authors ${ }^{4}$ have accepted the challenge of teasing apart the clinical effect/outcomes of UFH in a complicated cohort when using different laboratory testing to determine the "sweet spot" of therapy: minimal bleeding with no increase in thrombosis.

\section{References}

1. Monagle P, Chan A, Massicotte P, Chalmers E, Michelson AD. Antithrombotic therapy in children: the seventh ACCP conference on antithrombotic and thrombolytic therapy. Chest. 2004;126:645S-87S.

2. Smythe MA, Priziola J, Dobesh PP, Wirth D, Cuker A, Wittkowsky AK Guidance for the practical management of the heparin anticoagulants in the treatment of venous thromboembolism. J Thromb Thrombolysis. 2016;41:165-86.

3. Khaja WA, Bilen O, Lukner RB, Edwards R, Teruya J. Evaluation of heparin assay for coagulation management in newborns undergoing ECMO. Am J Clin Pathol. 2010;134:950-4.

4. Nair A, Oladunjoye O, Trenor C, LaRonde M, van den Bosch S, Sleeper L, et al. An anticoagulation protocol for use following 1 congenital cardiac surgery. J Thorac Cardiovasc Surg. 2018;156:343-52.e4.

5. Cuker A, Ptashkin B, Konkle BA, Pipe SW, Whinna HC, Xheng XL, et al. Interlaboratory agreement in the monitoring of unfractionated heparin using the anti-factor Xa-correlated activated partial thromboplastin time. J Thromb Haemost. 2009;7:80-6. 